\title{
The synchrotron mechanism and the high energy flare from PKS 1510-089
}

\author{
Z.N. Osmanov $* 1,2$ \\ ${ }^{1}$ School of Physics, Free University of Tbilisi, 0183, Tbilisi, Georgia \\ ${ }^{2}$ E. Kharadze Georgian National Astrophysical Observatory, Abastumani, 0301, Georgia
}

\begin{abstract}
In order to understand the role of the synchrotron emission in the high energy gamma-ray flares from PKS 1510-089, we study generation of the synchrotron emission by means of the feedback of cyclotron waves on the particle distribution via the diffusion process. The cyclotron resonance causes the diffusion of particles along and across the magnetic field lines. This process is described by the quasi-linear diffusion (QLD) that leads to the increase of pitch angles and generation of the synchrotron emission. We study the kinetic equation which defines the distribution of emitting particles. The redistribution is conditioned by two major factors, QLD and the dissipation process, that is caused by synchrotron reaction force. The QLD increases pitch angles, whereas the synchrotron force resists this process. The balance between these two forces guarantees the maintenance of the pitch angles and the corresponding synchrotron emission process. The model is analyzed for a wide range of physical parameters and it is shown that the mechanism of QLD provides the generation of high energy (HE) emission in the GeV energy domain. According to the model the lower energy, associated with the cyclotron modes, provokes the synchrotron radiation in the higher energy band.
\end{abstract}

Keywords: blazars: individual: PKS 1510-089 - radiation mechanisms: non-thermal-plasmas.

\section{Introduction}

During the last decade the observations in the high and the very high energy domains have stimulated theoretical studies of the corresponding astrophysical objects. Recently the AGILE (Astrorivelatore Gamma a Immagini LEggero) collaboration announced the discovery of the high energy (HE) $\gamma$-rays of PKS 1510-089 (D́Ammando et al., 2010), which is a nearby blazar $(z=0.361)$ belonging to the class of the flat radio quasars. The observations on the mentioned source was performed in the period 9 - 30 March 2009. According to the observational data, PKS 1510-089 showed extreme variability in $\gamma$-rays. It was found that the HE photon spectrum can be well described by the photon index $\Gamma=1.95 \pm 0.15$, the following average integral flux above $100 \mathrm{MeV},(311 \pm 21) \times 10^{-8}$ photons $\mathrm{cm}^{-2} \mathrm{~s}^{-1}$, and a peak integral flux $(702 \pm 131) \times 10^{-8}$ photons $\mathrm{cm}^{-2} \mathrm{~s}^{-1}$ detected on 25 of March (D́Ammando et al., 2010). The monitoring of the blazar between September 2008 and June 2009 was performed by the Fermi-LAT telescope and a complex variability at optical, UV and $\gamma$-ray bands was found (Abdo et al., 2010). During this period three $\gamma$-ray flares have been detected, with the brightest isotropic luminosity $2 \times 10^{48} \mathrm{erg} / \mathrm{s}$ on 2009 March 26 . It has been found that the flux for energies above $200 \mathrm{MeV}$ reaches its peak value $2.4 \times 10^{-7}$ photons ${ }^{-2} \mathrm{~s}^{-1}$.

Obviously it is assumed that the HE emission is produced by the inverse Compton mechanism (Blandford et al., 1990) or curvature radiation (Gangadhara, 1996, Thomas \& Gangadhara, 2005). The synchrotron process is supposed to be responsible only for producing relatively low energy photons, because in strong magnetic fields the cooling timescale is small compared with the kinematic timescale, that leads to the transition of relativistic electrons to their ground Landau states, resulting in the damping of the emission process.

*z.osmanov@freeuni.eduge 
Unlike the standard mechanism explaining the HE radiation, we apply the so-called quasi-linear diffusion (QLD) that prevents the pitch angles from damping, sustaining the synchrotron process. In particular, it is believed that plasmas in pulsar magnetospheres with strong magnetic fields may induce the unstable cyclotron waves (Kazbegi et al., 1991). These unstable modes in turn feedback on relativistic electrons and by means of the diffusion influence the particle distribution along and across the magnetic field lines (Lominadze et al., 1979, Machabeli \& Usov, 1979). Under certain conditions the physical system reaches the balance between the dissipation factors and the diffusion, leading to saturation of the pitch angles. Therefore, despite the efficient synchrotron losses, the pitch angles are maintained, which provides a continuous emission process.

The mechanism of QLD was successfully applied to pulsars (Chkheidze et al., 2007, 2011, Machabeli et 1., 2010, Machabeli \& Osmanov, 2010, Malov \& Machabeli, 2001), anomalous pulsars (Osmanov, 2021), the black hole located in the center of Milky Way, SgrA* (Gogaberishvili et al., 2021) and active galactic nuclei (AGN) (Osmanov, 2011, 2010, Osmanov \& Machabeli, 2010). In these papers it is shown that the QLD might provide the simultaneous generation of relatively low frequency waves and the HE $\gamma$-rays. On the other hand, it is observationally evident that some AGN reveal the strong correlation of HE and low frequency emission Bloom (2008), Giroletti et al. (2010), therefore the role of the QLD might be important for these sources. In particular, in Ref. Osmanov \& Machabeli (2010) the excitation of $X$-rays connected to the radio waves has been studied. The similar method was developed to examine the possibility of strong correlation of HE emission and submillimeter/infrared radiation (Osmanov, 2010) and study the generation of high and very high energy $\gamma$-rays strongly connected to radio emission (Osmanov, 2011).

Unlike the aforementioned articles, in the present work we focus on the concrete AGN. According to the observational data presented by AGILE collaboration (D́Ammando et al., 2010), the HE flare was detected during 9-30 March 2009 from blazar PKS 1510-089. We apply the mechanism of the QLD to the mentioned AGN and analyze the synchrotron radiation processes in producing the observed HE $(>100 \mathrm{MeV})$ photons. According to the standard theory of the synchrotron emission, it is assumed that the magnetic field is chaotic along the line of sight, therefore, in the framework of this approach the pitch angles vary in the broad interval, 0, $\pi$ (Ginzburg, 1981). Contrary to this scenario, the QLD prevents the pitch angles from damping, restricting their values.

The paper is arranged in the following way. In section II, we introduce the theory of the QLD. In section III, we apply the model to the blazar PKS 1510-089 and in section IV, we summarize our results.

\section{Main Consideration}

In this section we present our model and apply it to BL Lac PKS 0548-322. This is a low redshift blazar with the central supermassive black hole (SMBH) having the following mass $M \approx 5.4 \times 10^{8} M_{\odot}$ (Abdo et al., 2010), where $M_{\odot} \approx 2 \times 10^{33} \mathrm{~g}$ is the solar mass. Around the $\mathrm{SMBH}$, in a region of the lengthscale, $l \sim 10^{14-15} \mathrm{~cm}$, the magnetic field is strong enough to provide the frozen-in condition. In the magnetospheres of AGNs, Lorentz factors of the magnetospheric plasma particles lie in a broad interval, ranging from $\sim 1$ to $10^{8}$ (Osmanov et al., 2007, Rieger \& Aharonian, 2008). For simplicity we consider two component plasma: the relatively low energy electron-positron plasma component $\left(\gamma_{p}\right)$ and the highly relativistic electrons - the so-called beam component $\left(\gamma_{b}\right)$. As we have already mentioned, the relativistic particles will undergo strong synchrotron losses. In particular, the synchrotron cooling timescale for the beam electrons is given by the following expression $t_{\text {syn }} \sim$ $\gamma_{b} m c^{2} / P_{\text {syn }}$, where $m$ is the electron's mass, $c$ is the speed of light, $P_{\text {syn }} \approx 2 e^{4} \gamma_{b}^{2} B^{2} / 3 m^{2} c^{3}$ is the single particle synchrotron emission power, $e$ is the electron's charge and $B$ is the magnetic induction. By considering the relativistic electrons one can show that the synchrotron cooling timescale, $t_{c o o l}$, is of the order of $0.05 \times \gamma_{b 8}^{-2} B_{10}^{-2} \mathrm{~s}\left(\gamma_{b 8} \equiv \gamma_{b} / 10^{8}, B_{10} \equiv B / 10 G\right)$, whereas the kinematic timescale, $t_{k i n} \sim l / c \approx 3000 \times l_{14} \mathrm{~S}\left(l_{14} \equiv l / 10^{14} \mathrm{~cm}\right)$, for reasonable parameters is much smaller than $t_{\text {syn }}$. Moreover, the condition $t_{\text {cool }} / t_{\text {syn }} \ll 1$ becomes more strict for higher luminosity sources, or during $\gamma$-ray flares, since the equipartition magnetic field becomes higher in these cases. Therefore, due to the strong magnetic field, the synchrotron emission in the magnetosphere of PKS 1510-089 is strongly 
The synchrotron mechanism and the high energy flair from PKS 1510-089

suppressed by the energy losses and the particles rapidly transit to their ground Landau states and generation of radiation is stopped.

The situation drastically changes by means of the excited cyclotron waves. This problem was considered for pulsars by Kazbegi et al. (1991). In particular, since the magnetic field near the pulsar's surface is very strong, any transverse momenta of relativistic electrons are lost and the corresponding distribution function of electrons from the very beginning of motion becomes one-dimensional and anisotropic. Kazbegi et al. (1991) showed that for the aforementioned conditions the anomalous Doppler effect leads to the generation of the pure transversal cyclotron modes

$$
\omega_{t} \approx k c(1-\delta), \quad \delta=\frac{\omega_{p}^{2}}{4 \omega_{B}^{2} \gamma_{p}^{3}},
$$

where $k$ is the modulus of the wave vector, $\omega_{p} \equiv \sqrt{4 \pi n_{p} e^{2} / m}$ is the plasma frequency, $n_{p}$ is the plasma number density and $\omega_{B} \equiv e B / m c$ is the cyclotron frequency. We assume that energy in plasmas is distributed uniformly, $n_{p} \approx n_{b} \gamma_{b} / \gamma_{p}$ ( $n_{b}$ is the number density of the beam electrons). As it was shown in (Machabeli \& Usov, 1979) the mentioned cyclotron wave is characterized by the following frequency

$$
\nu \approx \frac{\omega_{B}}{2 \pi \delta \cdot \gamma_{b}} .
$$

The major requirement for excitation of the cyclotron modes is considerably strong magnetic field, so that the magnetic energy density, $W_{B}$, can exceed the plasma energy density, $W_{p}$. One can straightforwardly show that for the light cylinder lengthscales the mentioned condition $W_{B} / W_{p}>1$ writes as

$$
\frac{W_{B}}{W_{p}} \approx 100 \times \frac{10^{8}}{\gamma_{b}} \times \frac{100 \mathrm{~cm}^{-3}}{n_{b}}>1 .
$$

This in turn means that the maximum value of the beam number density, allowing the excitation of the cyclotron waves is of the order of $2 \times 10^{4} \mathrm{~cm}^{-3}$. If this condition is satisfied, the unstable cyclotron waves are excited, which by means of the feedback, through the diffusion process, affect the distribution of particles, creating the pitch angles.

On the other hand, since the emitting particles are extremely energetic they undergo the synchrotron radiation reaction force (Landau \& Lifshitz, 1971)

$$
F_{\perp}=-\alpha \frac{p_{\perp}}{p_{\|}}\left(1+\frac{p_{\perp}^{2}}{m^{2} c^{2}}\right), F_{\|}=-\frac{\alpha}{m^{2} c^{2}} p_{\perp}^{2} .
$$

Unlike the role of the diffusion, this force is responsible for the dissipation process, decreasing the pitch angle. Here $\alpha=2 e^{2} \omega_{B}^{2} / 3 c^{2}$ and $p_{\perp}$ and $p_{\|}$are transversal and longitudinal components of momentum respectively. The corresponding kinetic equation governing the mentioned mechanism is given in a series of works (Chkheidze et al., 2011)

$$
\begin{array}{r}
\frac{\partial f^{0}(\mathbf{p})}{\partial t}+\frac{\partial}{\partial p_{\|}}\left\{F_{\|} f^{0}(\mathbf{p})\right\}+\frac{1}{p_{\perp}} \frac{\partial}{\partial p_{\perp}}\left\{p_{\perp} F_{\perp} f^{0}(\mathbf{p})\right\}= \\
=\frac{1}{p_{\perp}} \frac{\partial}{\partial p_{\perp}}\left\{p_{\perp}\left(D_{\perp, \perp} \frac{\partial}{\partial p_{\perp}}+D_{\perp, \|} \frac{\partial}{\partial p_{\|}}\right) f^{0}(\mathbf{p})\right\}+ \\
+\frac{\partial}{\partial p_{\|}}\left\{\left(D_{\|, \perp} \frac{\partial}{\partial p_{\perp}}+D_{\|,\|} \frac{\partial}{\partial p_{\|}}\right) f^{0}(\mathbf{p})\right\},
\end{array}
$$

where $f^{0}(\mathbf{p})$ is the distribution function,

$$
\left(\begin{array}{c}
D_{\perp, \perp} \\
D_{\perp, \|}=D_{\| \perp} \\
D_{\|,\|}
\end{array}\right)=\left(\begin{array}{c}
D \delta\left|E_{k}\right|^{2} \\
-D \psi\left|E_{k}\right|^{2} \\
D \psi^{2} \frac{1}{\delta}\left|E_{k}\right|^{2}
\end{array}\right)
$$

are the diffusion coefficients (Chkheidze et al., 2011), $E_{k}$ is the electric field, square of which is given by $\left|E_{k}\right|^{2}=m c^{3} n_{b} \gamma_{b} /(4 \pi \nu)$ (Malov \& Machabeli, 2001), $\psi=p_{\|} / p_{\perp}$ is the pitch angle and $D=e^{2} / 8 c$. 
The synchrotron mechanism and the high energy flair from PKS 1510-089

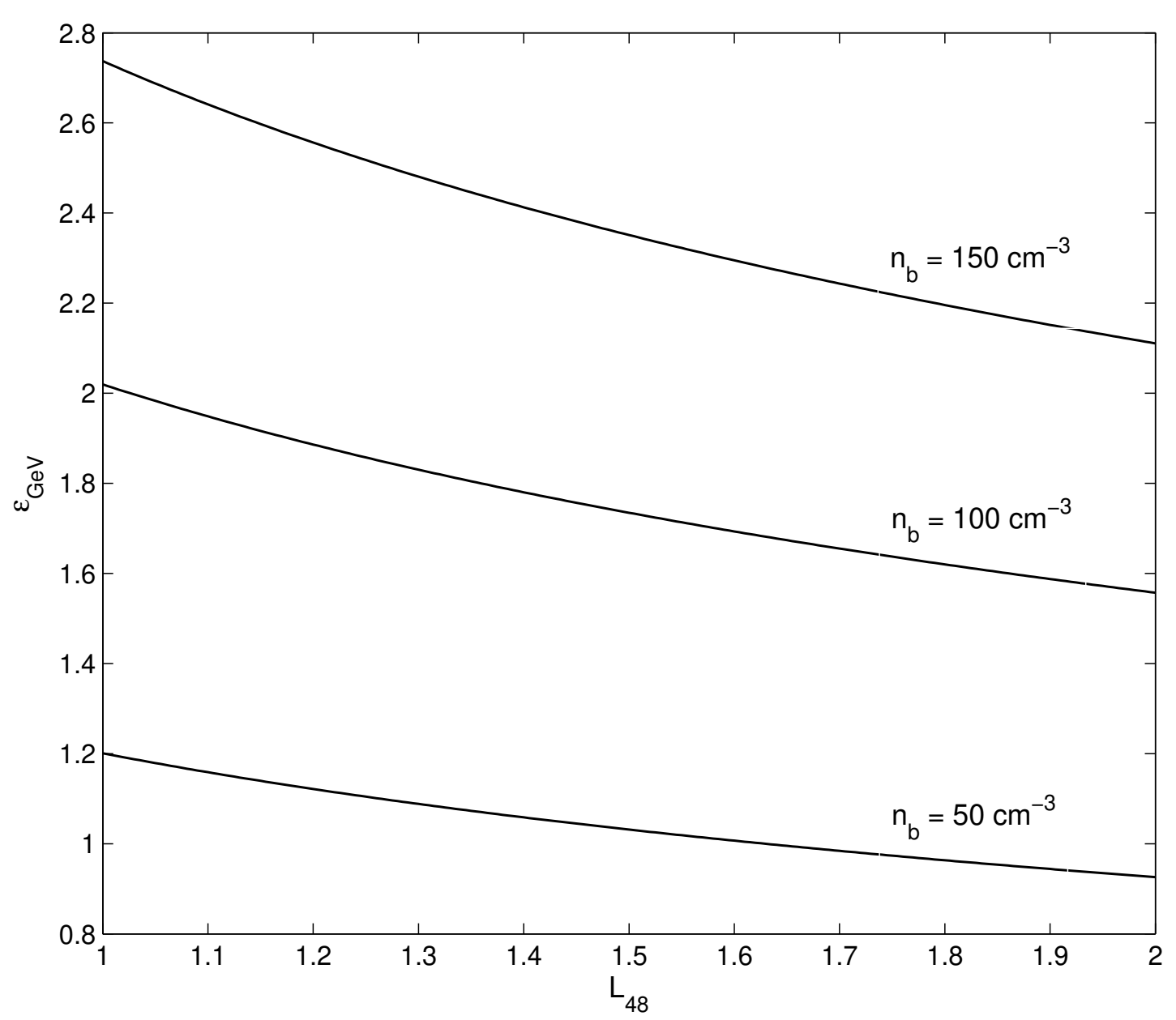

Figure 1. Behaviour of $\epsilon_{\mathrm{GeV}}$ with respect to the dimensionless luminosity $L_{48}$. The set of parameters is: $M \approx 5.4 \times 10^{8} M_{\odot}, r=70 R_{g}, n_{b} \in[50 ; 100 ; 150] \mathrm{cm}^{-3}, \gamma_{p} \approx 1$ and $\gamma_{b}=2 \times 10^{8}$.

Normally the pitch angles are very small, $\psi \ll 1$, therefore, one can reduce Eq. (5) by using the relation $\partial / \partial p_{\perp}>>\partial / \partial p_{\|}$(Chkheidze et al., 2011)

In the framework of the model the pitch angles saturate due to the balance between the diffusion and the dissipation factors. This leads to the stationary regime $(\partial / \partial t=0)$ and the solution of Eq. (9) writes as

$$
f\left(p_{\perp}\right)=C \exp \left(\int \frac{F_{\perp}}{D_{\perp, \perp}} d p_{\perp}\right)=C e^{-\left(\frac{p_{\perp}}{p_{\perp}}\right)^{4}}
$$

where

$$
\begin{gathered}
p_{\perp_{0}}=\left(\frac{4 \gamma_{b} m^{3} c^{3} D_{\perp, \perp}}{\alpha}\right)^{1 / 4} . \\
\frac{\partial f^{0}}{\partial t}+\frac{1}{p_{\perp}} \frac{\partial}{\partial p_{\perp}}\left(p_{\perp} F_{\perp} f^{0}\right)=\frac{1}{p_{\perp}} \frac{\partial}{\partial p_{\perp}}\left(p_{\perp} D_{\perp, \perp} \frac{\partial f^{0}}{\partial p_{\perp}}\right) .
\end{gathered}
$$

It is evident from Eq. (7) that only a tiny fraction of electrons have the transversal momentum sufficiently exceeding the value of $p_{\perp_{0}}$. Therefore, it is worth estimating the average value of $p_{\perp}$

$$
\left\langle p_{\perp}\right\rangle=\frac{\int_{0}^{\infty} p_{\perp} f\left(p_{\perp}\right) d p_{\perp}}{\int_{0}^{\infty} f\left(p_{\perp}\right) d p_{\perp}} \approx \frac{p_{\perp_{0}}}{2},
$$




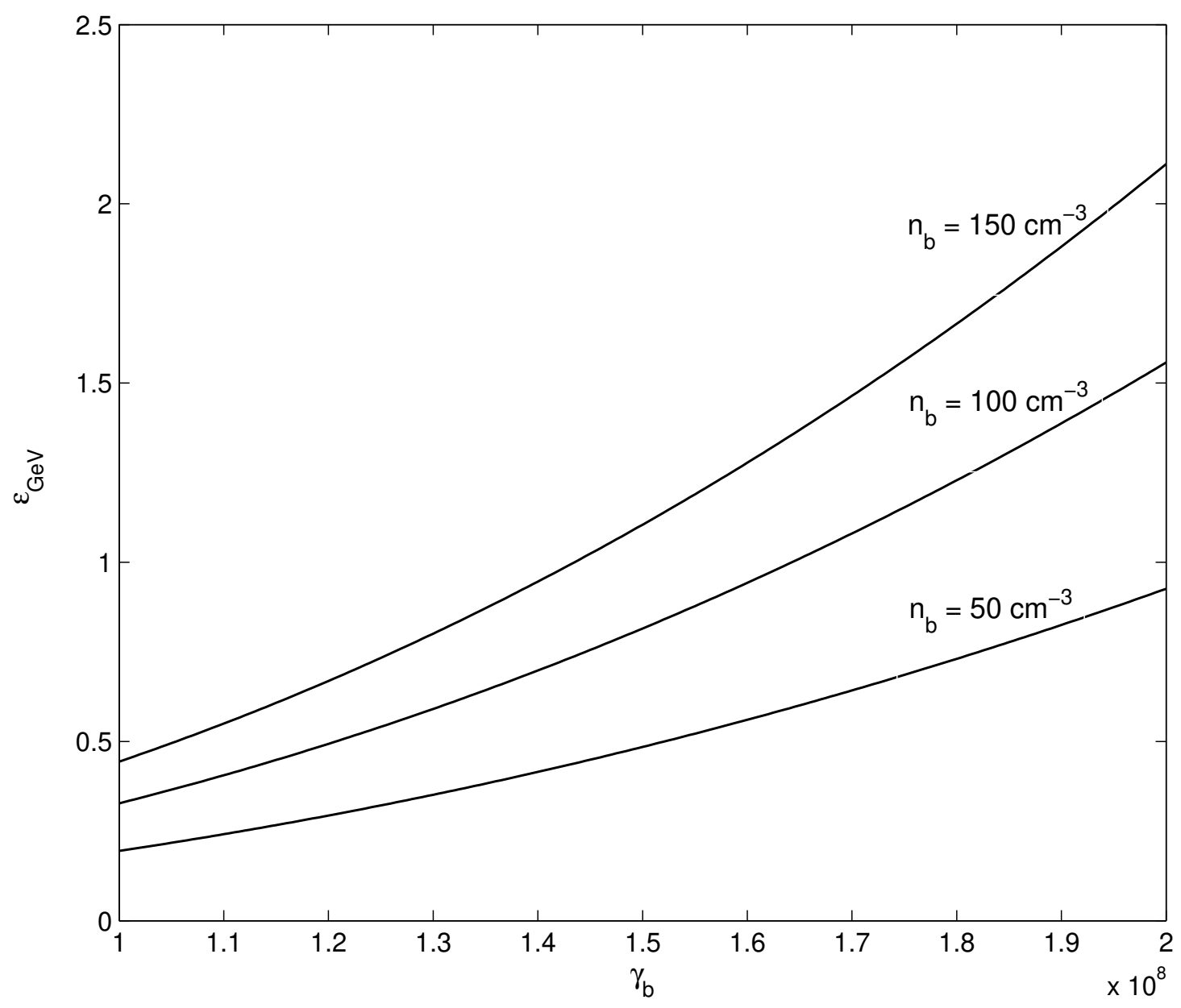

Figure 2. Behaviour of $\epsilon_{G e V}$ with respect to the Lorentz factor of relativistic electrons. The set of parameters is: $M \approx 5.4 \times 10^{8} M_{\odot}, r=70 R_{g}, n_{b} \in[50 ; 100 ; 150] \mathrm{cm}^{-3}, \gamma_{p} \approx 1, L=2 \times 10^{48} \mathrm{erg} / \mathrm{s}$.

which naturally describes the mean value of the pitch angles, $\langle\psi\rangle=\left\langle p_{\perp}\right\rangle / p_{\|}$, and the corresponding photon energy produced by the synchrotron emission (Rybicki \& Lightman, 1979)

$$
\epsilon_{M e V} \approx 2.5 \times 10^{-15} \frac{\gamma_{b} p_{\perp_{0}} B}{m c} .
$$

Generally speaking, strong magnetic field leads to efficient energy losses, resulting in one-dimensional distribution function. This in turn, creates all necessary conditions for excitation of the cyclotron waves, leading to the final result of this complex process: the creation of the pitch angles, that inevitably causes synchrotron radiation. Therefore, as it is clear from the consideration, the strong synchrotron energy losses do not impose any constraints.

\section{Discussion}

In this section we study properties of the HE emission of the blazar PKS 1510-089. According to Abdo et al. (2010), between September 2008 and June 2009 the Fermi LAT has detected three $\gamma$-ray flares. The most luminous isotropic flare with the estimated luminosity $7 \times 10^{47} \mathrm{erg} / \mathrm{s}$ was detected during 2009-03-10 and 2009-04-09, with the brightest daily luminosity of the order of $2 \times 10^{48} \mathrm{erg} / \mathrm{s}$ (March 29 2009). The equipartition magnetic field depends on the source luminosity, $B \approx \sqrt{2 L /\left(r^{2} c\right)}$ ( $r$ is the distance from blazar). Therefore, effectiveness of the QLD strongly depends on the flare efficiency, since the diffusion coefficient depends on $B$ (see Eq. 6). 


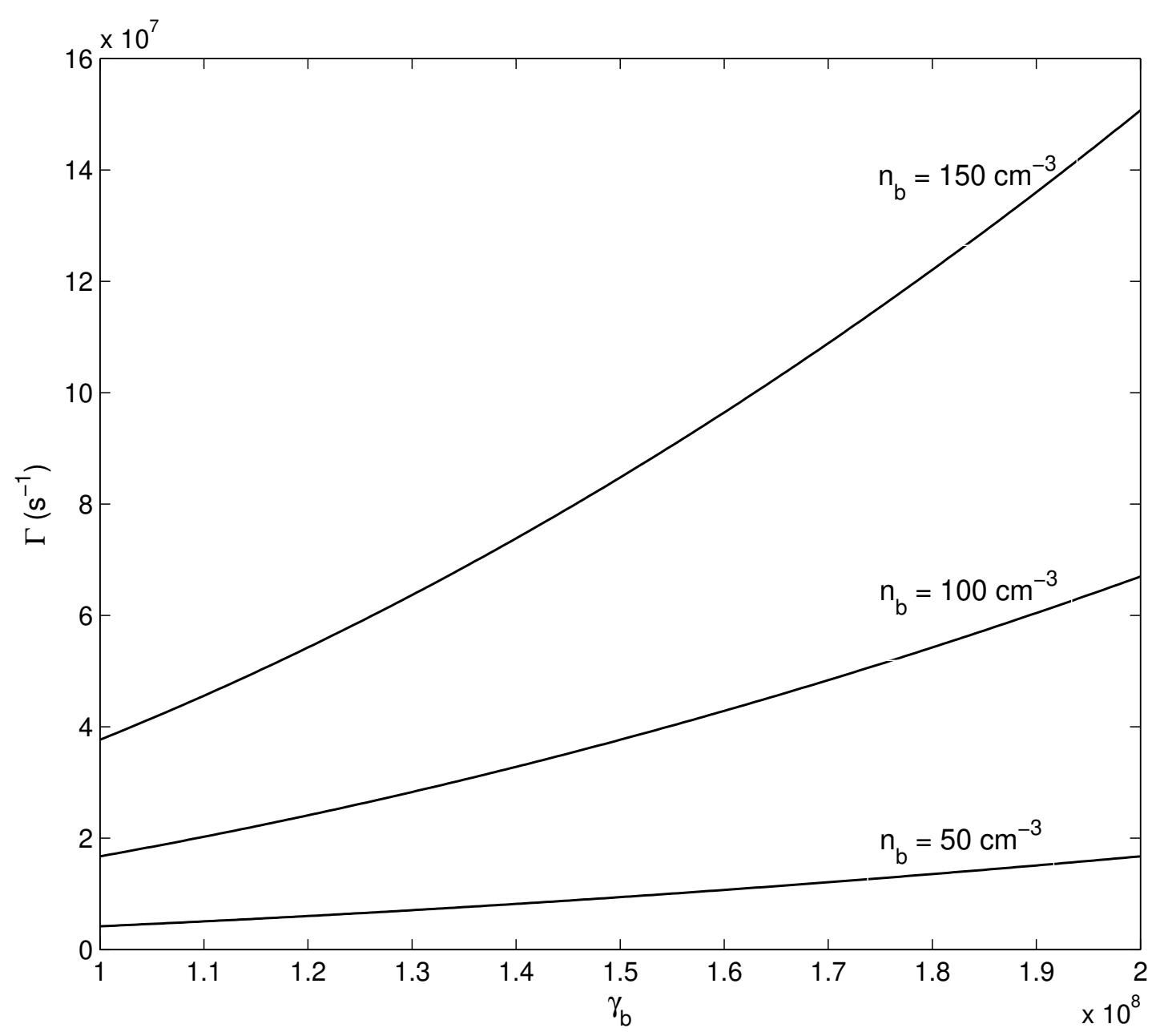

Figure 3. Behaviour of $\Gamma$ with respect to the Lorentz factor of relativistic electrons. The set of parameters is: $M \approx 5.4 \times 10^{8} M_{\odot}, r=70 R_{g}, n_{b} \in[50 ; 100 ; 150] \mathrm{cm}^{-3}, \gamma_{p} \approx 1, L=2 \times 10^{48} \mathrm{erg} / \mathrm{s}$.

In Fig. 1 we show the dependence of $\epsilon_{G e V}$ on the dimensionless luminosity, $L_{48} \equiv \frac{L}{10^{48} \mathrm{erg} / \mathrm{s}}$ for different densities of the beam electrons. The set of parameters is: $M \approx 5.4 \times 10^{8} M_{\odot}, r=70 R_{g}$, $n_{b} \in[50 ; 100 ; 150] \mathrm{cm}^{-3}, \gamma_{p} \approx 1$ and $\gamma_{b}=2 \times 10^{8}$. As it is evident from the figure, the photon energy is a continuously decreasing function of the source luminosity. This is a direct consequence of the following behaviour $p_{\perp_{0}} \propto L^{-5 / 8}$ (see Eq. 6), which means that for more luminous sources the pitch angles, $\langle\psi\rangle \propto p_{\perp_{0}}$, and the corresponding synchrotron photon energies will be lower. As it is clear from the plots, by increasing the density, the corresponding photon energy increases as well. In particular, by taking into account Eqs. (8-11) one can see that $\epsilon_{M e V} \propto n_{b}^{1 / 2}$. From the plots we see that the QLD may provide $\mathrm{HE}$ emission in the GeV energy domain if the beam electrons' Lorentz factors and number density are of the order of $2 \times 10^{8}$ and $50 ; 100 ; 150 \mathrm{~cm}^{-3}$, respectively and $\gamma_{p} \approx 1$.

In Fig. 2 we show the dependence of the photon energy on the Lorentz factors of the beam electrons. The set of parameters is the same as for Fig. 1, except $L=2 \times 10^{48} \mathrm{erg} / \mathrm{s}$ and $\gamma_{b} \in[1-2] \times 10^{8}$. As we see from the figures, the QLD may provide the synchrotron emission from multi MeV to several $\mathrm{GeV}$, which is in a good agreement with the observations (Abdo et al., 2010, D́Ammando et al., 2010). On the other hand, it is worth noting that according to the multifrequency campaigns, the spectral energy distribution (SED) of the blazar PKS 1510-089 shows two clear peaks, one in the GeV energy domain and another peak near $10^{13} \mathrm{~Hz}$. This particular peak is formed by mildly relativistic electrons in a different location, where magnetic induction is supposed to be of the order of $3 \mathrm{G}$. In particular, by assuming that the corresponding emission is produced by the synchrotron mechanism, one can straightforwardly show from $\nu_{1} \approx 2.9 \times 10^{6} \gamma^{2} \mathrm{BHz}$ (Rybicki \& Lightman, 1979) that the mildly 
relativistic electrons with $\gamma \sim 50$ can explain the first peak of SED. On the other hand, it is clear that energy losses in this case are not efficient, because $t_{\text {syn }} \sim 10^{6}$ s exceeds the kinematic timescale and hence the synchrotron process does not require any additional mechanism for maintaining the radiation. Therefore, according to our model, the HE emission comes from the nearby zone of the light cylinder surface $r \sim 5.6 \times 10^{15} \mathrm{~cm}$ (inner magnetosphere), whereas the first peak with $10^{13} \mathrm{~Hz}$ is formed in an outer magnetosphere of PKS 1510-089, where the magnetic field is lower.

Since the mechanism of the QLD is driven by the unstable cyclotron waves, it is important to investigate the effectiveness of the instability and the corresponding growth rate. Kazbegi et al. (1991) has shown that the increment of the cyclotron instability is given by

$$
\Gamma=\frac{\omega_{b}^{2}}{2 \nu \gamma_{p}}
$$

where $\omega_{b} \equiv \sqrt{4 \pi n_{b} e^{2} / m}$ is the plasma frequency of beam electrons. In Fig. 3 we show the behaviour $\Gamma\left(\gamma_{b}\right)$. The list of parameters is the same as for Fig. 2. As it is clear from the plots, depending on the physical parameters, the Growth rate varies in a broad interval $\left[5 \times 10^{6}-1.5 \times 10^{8}\right] \mathrm{s}^{-1}$, leading to the following timescales $t_{\text {ins }} \sim 1 / \Gamma \sim\left[7 \times 10^{-9}-2 \times 10^{-7}\right] \mathrm{s}$. On the other hand, the kinematic timescale $t_{k i n} \sim r / c$ is of the order of $2 \times 10^{5} \mathrm{~s}$, which exceeds that of the instability by many orders of magnitude. Therefore, the cyclotron instability is extremely efficient to provide the excitation of the cyclotron waves and maintain the synchrotron emission regime.

\section{Summary}

1) We study the recently detected (by AGILE and Fermi-LAT) HE $\gamma$-ray emission from the blazar PKS 1510-089. Applying the mechanism of the QLD we argue that in spite of the strong synchrotron energy losses, the diffusion is effective enough to provide the observed energies of the source.

2) It is shown that the excited unstable cyclotron waves strongly influence the distribution function of relativistic electrons by means of the quasi-linear diffusion. This in turn maintains the pitch angles from damping and provides the continuous synchrotron emission process.

3) We found that under favorable conditions the QLD may guarantee the HE emission with energies from multi $\mathrm{MeV}$ to several $\mathrm{GeV}$, that is in a good agreement with the observations of AGILE and Fermi-LAT.

As we see, the synchrotron mechanism may guarantee emission in the HE domain. Another important issue that we would like to address is the emission spectrum. As we have already mentioned in the introduction, according to the standard theory of the synchrotron radiation (Ginzburg, 1981) the magnetic field is chaotic along the line of sight, therefore, the corresponding values of the pitch angles lie in a broad interval, $\psi \in(0 ; \pi)$. Unlike this case, in the framework of the QLD the pitch angles are restricted by the dissipation factors leading to a certain spectral picture. Therefore, sooner or later we are going to examine this particular problem as well.

\section{Acknowledgments}

The author is grateful to Prof. George Machabeli for valuable discussions and helpful suggestions.

\section{References}

Abdo, A.A. et al. (Fermi collaboration), 2010, ApJ, 721, 1425

D́Ammando et al. (AGILE collaboration), 2010, A\&A, 521, 69

Blandford, R. D., Netzer, H., \& Woltjer, L. 1990, Active Galactic Nuclei, Springer-Verlag 
Bloom, S. D. 2008, AJ, 136, 1533

Chkheidze N. \& Machabeli G., 2007, A\&A, 471, 599

Chkheidze, N., Machabeli, G. \& Osmanov, Z., 2011, ApJ, 721, 318

Gangadhara, R. T. 1996, A\&A, 314, 853

Ginzburg, V. L. 1981, "Teor. Fizika i Astrofizika", Nauka M. 1981

Giroletti, M., Reimer, A., Fuhrmann, L. et al. 2010, [arXiv:astro-ph/1001.5123]

Gogaberishvili, G., Osmanov, Z.N. \& Mahajan S.M., 2021, MNRAS, 503, 3592

Hartman, R. C., et al. 1999, ApJS, 123, 79

Kazbegi, A. Z., Machabeli, G. Z., \& Melikidze, G. I. 1991, MNRAS, 253, 377

Landau, L. D., \& Lifshitz, E. M. 1971, Classical Theory of Fields (London: Pergamon)

Lominadze J.G., Machabeli G.Z. \& Mikhailovsky A.B., 1979, J. Phys. Colloq., 40, No. C-7, 713

Machabeli G. \& Osmanov Z., 2009, ApJL, 700, 114

Machabeli G. \& Osmanov Z., 2010, ApJ, 709, 547

Machabeli G.Z. \& Usov V.V., 1979, AZhh Pis'ma, 5, 445

Malov I.F. \& Machabeli G.Z., 2001, ApJ, 554, 587

Osmanov, Z.N., 2021, Com. BAO, 68, 114

Osmanov, Z., 2011, A\&A, 530, 75

Osmanov, Z., 2010, ApJ, 721, 318

Osmanov Z. \& Machabeli G., 2010, A\&A, 516, A12

Osmanov, Z., Rogava, A. D., \& Bodo, G. 2007, A\&A, 470, 395

Rieger, F. M., \& Aharonian, F. A. 2008, A\&A, 479, L5

Rybicki, G.B. \& Lightman, A. P., 1979, Radiative Processes in Astrophysics. Wiley, New York

Thomas, R. M. C., \& Gangadhara, R. T. 2005, A\&A, 437, 537 